\title{
Tensor Velocity Imaging with Motion Correction
}

\author{
Jørgensen, Lasse Thurmann; Schou, Mikkel; Stuart, Matthias Bo; Jensen, Jørgen Arendt
}

Published in:

IEEE Transactions on Ultrasonics, Ferroelectrics, and Frequency Control

Link to article, DOI:

10.1109/TUFFC.2020.3046101

Publication date:

2021

Document Version

Peer reviewed version

Link back to DTU Orbit

Citation (APA):

Jørgensen, L. T., Schou, M., Stuart, M. B., \& Jensen, J. A. (2021). Tensor Velocity Imaging with Motion Correction. IEEE Transactions on Ultrasonics, Ferroelectrics, and Frequency Control, 68(5), 1676-1686 . https://doi.org/10.1109/TUFFC.2020.3046101

\section{General rights}

Copyright and moral rights for the publications made accessible in the public portal are retained by the authors and/or other copyright owners and it is a condition of accessing publications that users recognise and abide by the legal requirements associated with these rights.

- Users may download and print one copy of any publication from the public portal for the purpose of private study or research.

- You may not further distribute the material or use it for any profit-making activity or commercial gain

- You may freely distribute the URL identifying the publication in the public portal 


\title{
Tensor Velocity Imaging with Motion Correction
}

\author{
Lasse Thurmann Jørgensen, Mikkel Schou, Matthias Bo Stuart, Jørgen Arendt Jensen \\ Center for Fast Ultrasound Imaging, Department of Health Technology, Technical University of Denmark, \\ DK-2800 Lyngby, Denmark
}

\begin{abstract}
This paper presents a motion compensation procedure that significantly improves the accuracy of synthetic aperture tensor velocity estimates for row-column arrays. The proposed motion compensation scheme reduces motion effects by moving the image coordinates with the velocity field during summation of low-resolution volumes. The velocity field is estimated using a transverse oscillation cross-correlation estimator and each image coordinate's local tensor velocity is determined by upsampling the field using spline interpolation. The motion compensation procedure is validated using Field II simulations and flow measurements acquired using a $3 \mathrm{MHz}$ row-column addressed probe and the research scanner SARUS. For a peak velocity of $25 \mathrm{~cm} / \mathrm{s}$, a pulse repetition frequency of $2 \mathrm{kHz}$ and a beam-to-flow angle of 60 degrees, the proposed motion compensation procedure was able to reduce the relative bias from $-27.0 \%$ to $-9.4 \%$ and standard deviation from $8.6 \%$ to $8.1 \%$. In simulations performed with a pulse repetition frequency of $\mathbf{1 0}$ $\mathrm{kHz}$, the proposed method reduces the bias in all cases with beam-to-flow angles of 60 and 75 degrees and peak velocities between 10 and $150 \mathrm{~cm} / \mathrm{s}$.
\end{abstract}

Index Terms-Motion correction, Tensor velocity imaging, Row column addressed probes, volumetric imaging.

\section{INTRODUCTION}

Ultrasonic vector flow imaging (VFI) is a technique that estimates the flow's angle and magnitude. It is an improvement to the traditional velocity estimation approaches that only estimates velocity along the direction of the emitted ultrasound beam, i.e., the axial direction. VFI is, thus, less user-depended and requires less interpretation of the results when compared to the traditional approach. Several methods have been demonstrated to perform 2-D VFI: Multi-beam methods uses two or more beams with different incident angles to derive the velocity components of the flow [1]. Speckle tracking uses the sum of differences or cross-correlation to trace speckle patterns between frames [2]. The transverse oscillation (TO) method, developed by Jensen and Munk [3], relies on generating oscillations transverse to the beam direction to increase transverse motion sensitivity. However, 2-D VFI still only shows a projection of the complex 3-D flow on a 2-D plane. It, therefore, fails to capture details of the flow that could potentially disclose abnormal vascular conditions. For example, in [4], 2-D VFI is used to examine the flow in the ascending aorta, and because the technique cannot capture out-of-plane motion, the cross-sectional view of flow is seen as a 2-D whirl. However, the flow is more likely helicoidal, which is a flow structure 2-D VFI cannot capture.

VFI, in its most ideal form, would be able to show a 3-D velocity vector at each point in the volume at high frame rates. This ideal form of VFI is in this work called tensor velocity imaging (TVI). The benefit of a complete flow dataset is that no assumptions about the 3-D flow geometry are needed. This can improve blood volume flow estimations, which in 2-D systems is based on estimating the vascular cross-sectional area [5]. It can also improve pressure gradient estimates, which are derived in the current approach, assuming a negligible outof-plane motion [6].

Extending the 2-D VFI estimators to perform TVI requires fast volumetric imaging, which can be achieved with 2-D probes. The first 2-D probes were introduced in the early 1990s [7], [8], where it was proposed to address the elements in a matrix grid. However, maintaining a low F-number for all imaging depth requires 128 to 192 elements in the matrix's side length, which translates to $192^{2}=36,864$ independent elements and channels. A probe with such a channel count would be practically impossible to make and yield a broad and heavy cable with data rates in the hundreds of gigabytes per second. Different design solutions have been proposed to overcome the dimensionality issue of the fully addressed matrix probe [7], [9]-[13].

One solution is to use sparse 2-D arrays. Here, the surface area of the fully addressed array is covered using a subset of the channels. The approach allows for one-to-one element channel connectivity, and thus preserves the steering flexibility of the fully addressed matrix arrays. However, the reduction of the active surface area can decrease the signalto-noise ratio and increase the overall side-lobe energy [14], [15]. Other techniques such as micro-beamforming and rowcolumn addressing allow for a full active surface area with the trade-off being a loss of the one-to-one element channel correspondence, and, thus, a loss of the steering flexibility.

Channel reduction is achieved with micro-beamforming by adding, in-probe, the response from neighboring channels with a specified steering delay. With this solution, grating lobes are reduced as long as the delay profile in the delay-and-sum beamformation roughly matches with the delay applied in the in-probe summations [16]. Consequently, each emission's field of view (FoV) is effectively limited, and multiple emissions may be required to fully cover the region-of-interest.

Row-column addressed arrays (RCAs) reduce the number of channels from $N^{2}$ to $2 N$ by addressing the elements of the 2-D array by rows and columns. Thus, RCAs only use a fraction $(2 / N)$ of the matrix probe's channels, which makes it more feasible to achieve a low F-number at large image depth. However, the FoV is restricted to the volume directly below the aperture surface for a flat-surfaced probe, and multiple emissions are required to obtain a 3-D resolution in the full volume. 
Though each of the mentioned 2-D probe types has to various extents shown capabilities in volumetric flow visualization [17]-[25], this work focuses solely on RCAs as this design solution offers the most efficient channel reduction and is thus, from a fabrication and processing standpoint the least complex approach to volumetric imaging.

RCAs were first suggested by Morton and Lockwood in 2003 [11] and is effectively two overlaid, orthogonal 1-D probes, where one emits, and the other receives the backscattered signal. In [24] 3-D flow estimations along a flow-line was performed using an RCA probe. The approach presented acquires the 3-D velocity estimates line-by-line and has, therefore, a restricted volumetric coverage within a specific time frame. Improvements to the volumetric coverage at high frame rates has been achieved using synthetic aperture (SA) imaging and plane wave imaging [19], [23]. Here, the RCA resolves the entire image volume through an SA emissions sequence, which isonifies the volume from different angles with defocused emissions. A low-resolution volume (LRV) is beamformed from each emission, and combining all LRVs from each angle establishes dynamic transmit focus, since the LRVs sums in phase at the position of the scatterers. The combined LRVs create a high-resolution volume (HRV), which is dynamically focused both in transmit and receive. The LRVs are, however, only compounded coherently, if the scatterers are stationary during the acquisition period of the HRV. Phase misalignment occurs if the imaged object is rapidly moving, as can be the case for blood flow. Other researchers have studied the motion effects on SA systems [26]-[34], and lateral motion is reported to cause a broadening of the point spread function (PSF), and axial motion is found to cause a spatial shift of the PSF [26]. The latter is far more detrimental as it causes a change in the geometry of the moving object. This was also the case in [23], where axial motion introduced a considerable reduction in the TVI estimation accuracy.

The motion artifact can be reduced by correcting for motion before compounding [33], [34]. Here, motion is estimated at each image point before subsequently registering the lowresolution images to reduce the inter-frame motion. Thus, the motion-corrected low-resolution images yield a highresolution image with an improved PSF due to the reduction in motion effects. The motion correction has also been shown to increase the performance of velocity estimators in 2-D SA imaging systems: Oddershede and Jensen [32] demonstrated that 2-D motion compensation could significantly improve the bias and standard deviation of the 2-D VFI estimator, and Ekroll et. al. [35] achieved a similar reduction in the bias for the coherent compounding Doppler estimator when compensating for 2-D motion.

This work investigates the performance of the tensor velocity estimator when applying motion correction to the LRVs. The proposed procedure is applied and validated on RCA acquired data but applies in principle to all volumetric SA imaging systems capable of performing TVI. The procedure has been presented in previous work [36]; however, this work provides a more in-depth analysis of the approach through simulations and additional flow measurements to investigate the performance at a broader range of beam-to-flow angles, peak velocities, and iterations of the motion compensation algorithm.

The rest of the paper is organized as follows; in Section II, the RCA SA imaging is described in detail to provide the necessary framework for describing both the motion-effects (Section III) and the motion compensation algorithm (Section IV). Section V explains the implemented tensor velocity estimator, Section VI describes the SA emission sequence used for acquiring the flow data, and Section VII details the experimental setup as well as the validation process. Lastly, in Section VIII and IX, the results are presented and interpreted.

\section{ROW COLUMN IMAGING}

RCA transducers can be viewed as two stacked orthogonal 1-D linear arrays, where one isonifies the volume, and the other receives the back-scattered signal. This concept extends to the RCA delay-and-sum beamformer [37], which essentially is identical to the delay-and-sum algorithm for 1-D arrays [38] apart from the time-of-flight (ToF) calculations being performed in two 2-D domains; the $x z$-domain and the $y z$ domain. The two domains are referred to as the "transmit domain" and "receive domain." The orientations of the transmit and receive domain depend on which aperture emits and receives the signal. The column aperture is defined as having elements along the $x$-direction, and the row aperture as having elements along $y$. When the column aperture emits, and the row aperture receives, the transmit domain is the $x z$-domain, and the receive domain is the $y z$-domain. In the opposite case, $x$ and $y$ are interchanged. The ToF assuming that the column aperture emits and the row aperture receives is [37]:

$$
t_{t o f, i, j}(\vec{p})=\frac{d_{t}+d_{r}}{c},
$$

where

$$
\begin{gathered}
d_{r}=\sqrt{\left(y_{p}-y_{e, i}\right)^{2}+\left(z_{p}-z_{e, i}\right)^{2}}, \\
d_{t}= \begin{cases}z_{v, j}-r & \text { if } z_{p}<z_{v, j} \\
z_{v, j}+r & \text { otherwise }\end{cases}
\end{gathered}
$$

and

$$
r=\sqrt{\left(x_{p}-x_{v, j}\right)^{2}+\left(z_{p}-z_{v, j}\right)^{2}} .
$$

Here, $c$ denotes the speed of sound in the medium, $\vec{p}=$ $\left(x_{p}, y_{p}, z_{p}\right)$ is the position of the image point, $\left(x_{v, j}, z_{v, j}\right)$ is the $j$ th virtual source line (VSL) coordinate, and $\left(y_{e, i}, z_{e, i}\right)$ is the coordinate of the $i$ th receiving element. The virtual sources and the elements are modeled as line segments since a point in 2-D space is a line in 3-D space. The square root term in (2) and (3) express the radii of two orthogonal cylinders originating from $\left(x_{v, j}, z_{v, j}\right)$ and $\left(y_{p}, z_{p}\right)$ and models the wavefront from the emitted and received sound source. Note that the domain of (2) and (3) is, respectively, the transmit- and receive domain. Using (1) the beamformed image points of the LRV are given by:

$$
L R V_{j}(\vec{p})=\sum_{i=1}^{N} a_{r}(\vec{p}, i) s\left(t_{t o f, i, j}(\vec{p}), i\right),
$$




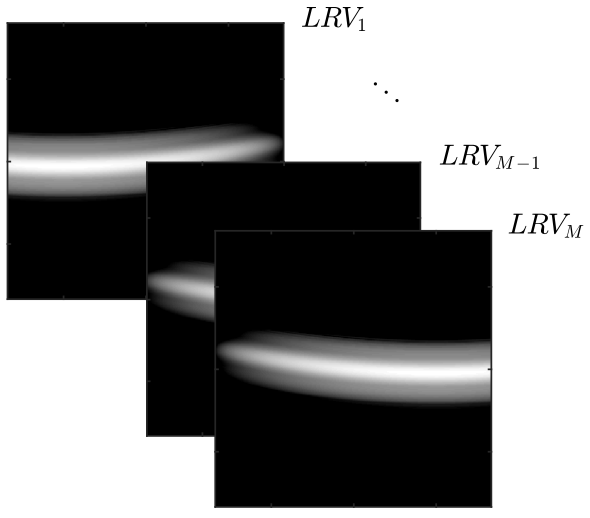

Summation

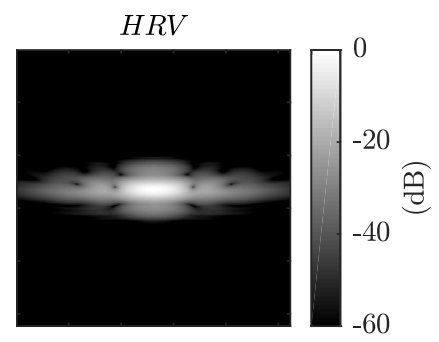

Fig. 1. Formation of the HRV. LRVs from different virtual sources are summed to create an HRV. The HRV shows a PSF of a point target at the center of the volume. Note that the LRVs cannot resolve this point target laterally.

where $a_{r}$ is receive element apodization, $N$ is the number of elements in receive, and $s(t, i)$ denotes the RF signal from the $i$ th channel at the measurement time $t$. The HRV is formed by summing the LRVs from the different VSL emissions:

$$
H R V(\vec{p})=\sum_{j=1}^{M} a_{v}(\vec{p}, j) L R V_{j}(\vec{p}),
$$

where $a_{v}$ denotes the apodization of the virtual source and $M$ is the number of VSLs. Fig. 1 illustrates the formation of the HRV from the summation of the LRVs. The HRV shows the PSF of a point target in the center of the volume. The LRVs cannot, individually, resolve the point target laterally, but lateral resolution can be obtained by summing the LRVs from the different VSL emissions. This is because the LRVs add coherently at the position of the point target and incoherently away from the point target.

\section{MOTION EFFECTS}

The effects of motion in RCA SA systems are best understood by first examining the structures of the LRV. Fig. 2 shows the $y=0$ plane in a LRV with the PSF for a point target at $\vec{p}=(0,0,20) \mathrm{mm}$. As the figure shows, the PSF cannot be resolved in the LRV and only curves of approximately constant amplitude along $x$ can be seen across the plane. The amplitude is approximately constant because the ToF along the position

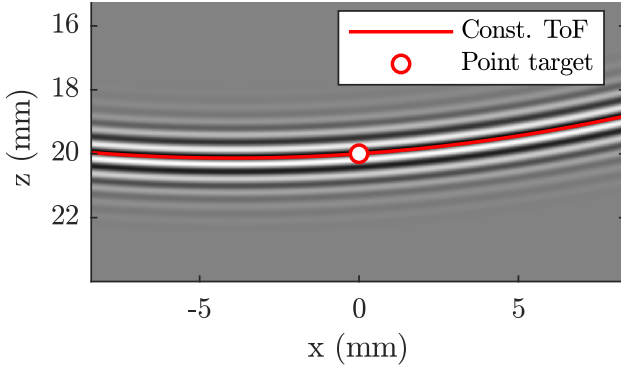

Fig. 2. LRV PSF in linear scale space. The imaging scheme cannot resolve the point target from a single LRV, and there is an approximately constant value along curves across the cross-section. The curve intersecting the point target is colored red.

of the curves are approximately constant. The positions of this constant ToF curve can be modeled by

$$
f_{j}(x, \vec{p})= \begin{cases}\frac{g}{2} & \text { if } x_{p}=x_{v, j} \\ \frac{g\left(g-2 z_{v, j}\right)-\left(x_{v, j}-x\right)^{2}}{2\left(g-2 z_{v, j}\right)} & \text { otherwhise }\end{cases}
$$

where

$$
g= \begin{cases}z_{v, j}+z_{p}-r & \text { if } z_{p}<z_{v, j} \\ z_{v, j}+z_{p}+r & \text { otherwise }\end{cases}
$$

The constant ToF curves can help visualize the effects of motion by letting $\vec{p}$ equal to the point targets' position and by subsequently moving it between emissions. Fig. 3 shows curves, calculated using (7), from $M$ emissions sources with a linear angle sequence [33]. The lines intersect the point target, which is static in the top image and moving in the middle and bottom image. When there is no motion, the lines intersect each other at the exact position of the point target. This intersection point is where the LRVs will add coherently and form the peak of the PSF in the HRV. When axial motion is introduced, the intersection point shifts along the $x$-axis. This creates a velocity depended offset in the HRV, which reduces the accuracy of the velocity estimation. The lateral shift does not occur when the motion is lateral. Here the lines' intersections are spread symmetrically around the average position of the point target. This widens the PSF, resulting in a blurring effect. The blurring has less of an impact on the estimator as the blurred HRVs can still be correlated. The distortion caused by axial motion is more detrimental, as it changes the geometry of the imaged moving tissue. From an acquisition standpoint, there are two overt ways to lessen the shift caused by the axial motion. One is to increase the pulse repetition frequency $\left(f_{p r f}\right)$, and the other is to increase the angle difference between emissions. However, increasing these parameters comes at a cost: a large angle difference can cause grating-lobes, and the $f_{p r f}$ inversely restricts imaging depth. It has also been suggested to use an alternated polarity sequence to mitigate the shift [33]. However, this approach is not recommended in RCA imaging as it effectively creates two opposite directed shifts, both roughly twice as large as the shift seen in the linear angle sequence. From a processing standpoint, the shift can be removed by moving the LRVs with 

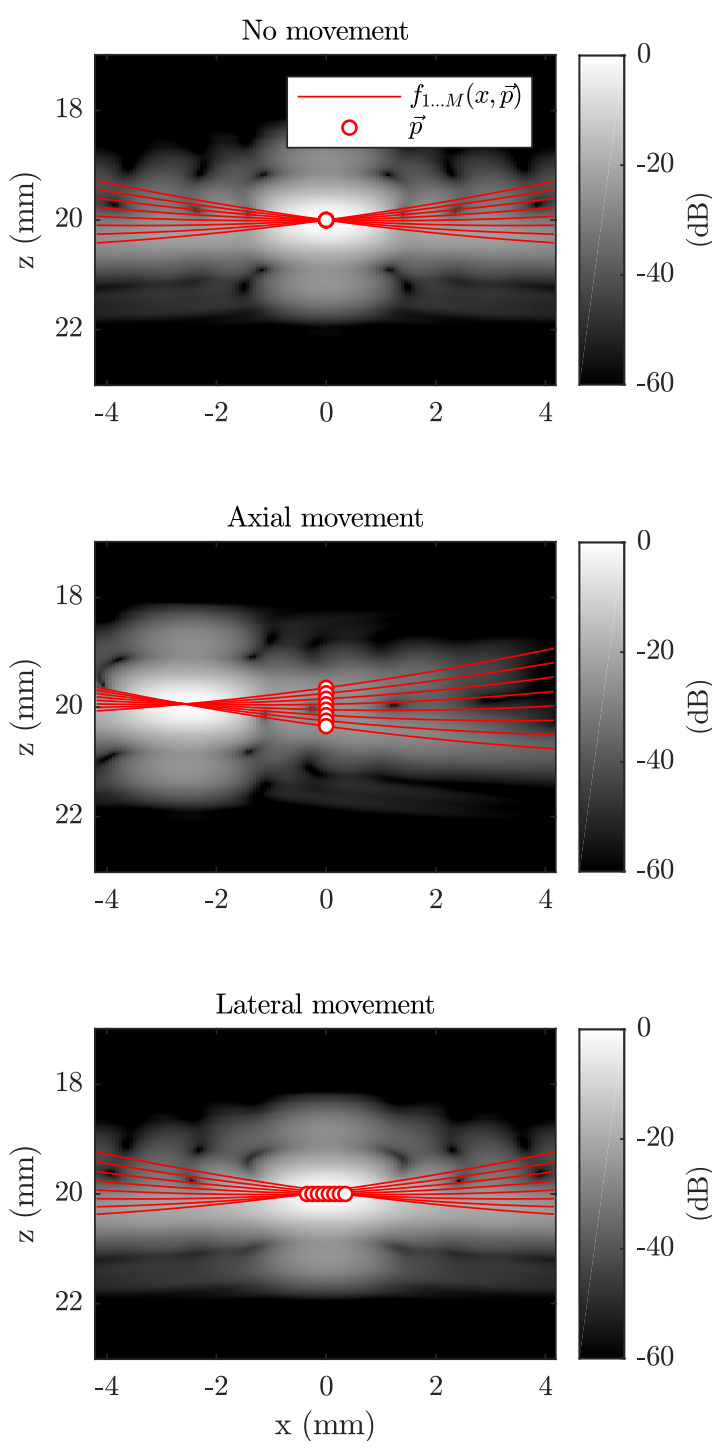

Fig. 3. Constant ToF lines (7) from $M$ emission sources shown on top of the resulting HRV PSF. The lines intersect a point target at $\vec{p}$, and the output is shown when the point target is without motion (top), moves axially (middle), and moves laterally (bottom).

the same velocity as the point target. The point target is, thus, kept static in the moving frame of reference.

\section{Motion COMPENSATION}

The motion compensation procedure is applied by moving the image coordinates using the local estimated velocity, $\vec{v}(\vec{p})=\left(v_{x}, v_{y}, v_{z}\right)(\vec{p})$, during the formation of the HRV. The coordinate translation aligns the center LRV for deformation and is given by:

$$
\Delta \vec{d}_{j}(\vec{p})=(j-(M+1) / 2) \frac{\vec{v}(\vec{p})}{f_{p r f}} .
$$

The HRV with reduced motion is then given by

$$
H R V\left(\vec{p}_{d}\right)=\sum_{j=1}^{M} a_{v}\left(\vec{p}_{d}, j\right) L R V_{j}\left(\vec{p}_{d}\right) .
$$

Where $\vec{p}_{d}=\vec{p}+\Delta \vec{d}_{j}(\vec{p})$. Note that the velocity term in (9) is independent of $j$ and thus assumes constant velocity throughout the summation period of the HRV. Fig. 4 illustrates the coordinates' translation between emissions. The figure shows how the grid coordinates are deformed to compensate for the motion. The LRV half-way through the summation period has no translation (assuming $M$ is an odd number). The other grid coordinates are then translated using (9), yielding an HRV with reduced motion effects.

A motion-corrected velocity estimator is inherently a recursive problem, as it relies on motion estimates to correct the motion effects. The proposed motion correction estimator can be expressed from (9) by extending the velocity term. Let $\vec{v}_{k}(\vec{p})$ denote the $k$ th iteration of the velocity vector. When performing the motion compensation the velocity term in (9) is given by the average velocity estimate across iterations:

$$
\bar{v}_{k}=\frac{1}{k-1} \sum_{i=1}^{k-1} \vec{v}_{i} \quad \text { for } k=2,3 \ldots
$$

The reason for using the average estimation is to promote a stable convergence. Had just the previous estimation been used, there could arise a situation, where the skewing caused by axial motion (see e.g., Section III) would cyclically reappear.

\section{TENSOR VELOCITY ESTIMATOR}

TVI is performed using the directional transverse oscillation (DTO) cross-correlation estimator [23], [39]. The approach uses directional beamforming in the three cartesian directions to estimate each direction's velocity component. The axial velocity can be estimated directly from the beamformed axial lines using cross-correlation. This is because the emitted field is already sensitive to axial motion, due to its inherent axial frequency component. This is not the case when estimating the transverse velocity, and thus a double peak apodization is applied in receive to generate a transverse oscillating field. The double peak apodization creates a field which oscillates laterally with the wavelength [3]:

$$
\lambda_{l a t}=2 \lambda \frac{z}{D}
$$

where $\lambda$ is the wavelength of the emitted pulse, and $D$ is the distance between the peaks. However, the field still contains the axial frequency component, and rapid de-correlation between the frames can occur, when axial motion is present. The de-correlation can be mitigated by filtering out the axial frequency component. This work uses a method described in [39], which reduces the axial frequency by combining outputs from a cascade of the Hilbert transform in the axial and transverse direction. Because the transverse oscillation is only induced in the receive domain, the sequence must be repeated with the receiving and transmitting aperture interchanged to estimate all three velocity components.

The unbiased cross-correlation function is used to estimate the velocity components and is for two consecutive signals $r_{1}(n)$ and $r_{2}(n)$ in $\mathbb{C}^{N}$ defined as

$$
R_{12}(m)=\frac{\sum_{n=-\infty}^{\infty} r_{1}^{*}(n) r_{2}(n+m)}{|N-m|},
$$



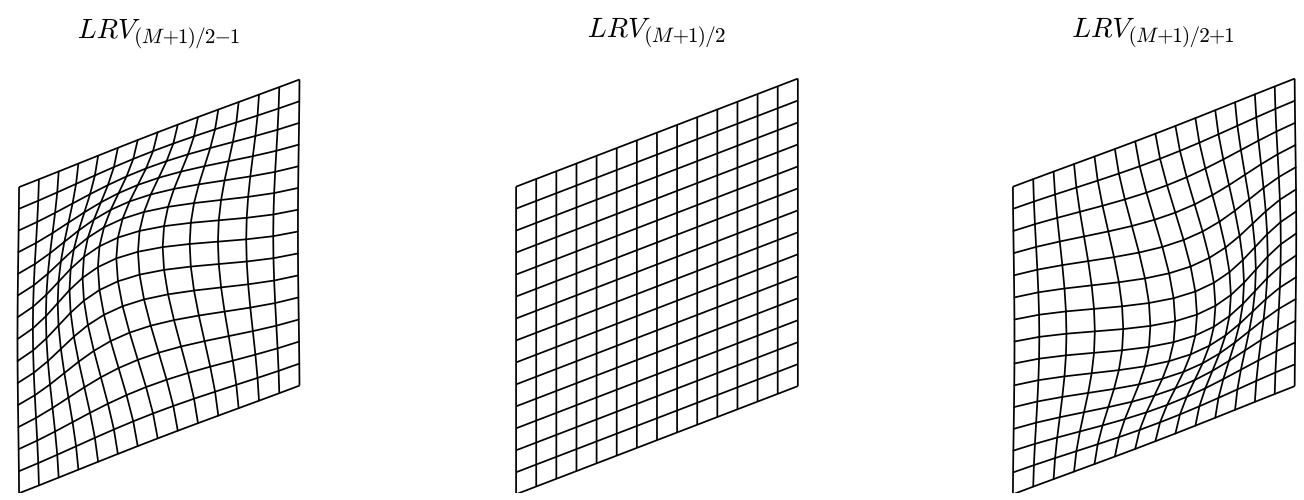

Fig. 4. LRV image grid deformation during motion compensation. For each emission, the grid coordinates are translated based on the local velocity estimation and the pulse repetition period.

where $r_{1}^{*}(n)$ is the complex conjugate of $r_{1}(n)$ and $m$ denotes the lag. The lag at the peak indicates the displacement between the two signals. The real part of the correlation function is used because it has been found to achieve a more precise result [39]. The displacement lag, $m_{p}$, is made into a continuous metric by fitting a second-order polynomial to the peak and its neighboring points. The continuous displacement lag is then calculated as the peak of the polynomial using [40]:

$$
m_{p q}=m_{p}-\frac{R_{12}\left(m_{p}+1\right)-R_{12}\left(m_{p}-1\right)}{R_{12}\left(m_{p}+1\right)-2 R_{12}\left(m_{p}\right)+R_{12}\left(m_{p}-1\right)},
$$

and converted to velocity using:

$$
v=m_{p q} \frac{c f_{p r f, e f f}}{2 f_{s}},
$$

where $f_{s}$ denotes the sampling rate, $c$ is the speed of sound in the medium, and $1 / f_{\text {prf,eff }}$ is the effective pulse repetition period, which is the duration between $r_{1}(n)$ and $r_{2}(n)$.

Several measures are taken to reduce the probability of detecting an erroneous correlation peak. The first is an upper limit for the displacement. The maximum displacement is chosen based on the highest velocity expected, $v_{\max }$, and is calculated as

$$
\pm m_{\max }= \pm\left\lceil v_{\max } \frac{2 f_{s}}{c f_{\text {prf }, \text { eff }}}\right\rceil,
$$

where the brackets denote the ceiling function. The second measure is averaging several consecutive cross-correlations and cross-correlations from neighboring segments. The averaged cross-correlations are normalized before summation to prevent a small subset from determining the estimation. The cross-correlation is normalized to the auto-correlations in lag zero, which ensures the cross-correlation coefficients are within a range of \pm 1 . The averaged and normalized crosscorrelation function is defined as:

$$
\hat{R}_{12}(m)=\sum_{i=1}^{L} \frac{R_{12, i}(m)}{\sqrt{\left(R_{11, i}(0) R_{22, i}(0)\right)}},
$$

where $i$ denotes the index of the $L$ realizations being averaged. In this work, $9 \times 9$ neighbouring correlation were averaged corresponding to an area of $(2 \lambda)^{2}$ when estimating lateral velocities

16 consecutive cross-correlation functions were used per velocity estimate, which at $f_{p r f}=10 \mathrm{kHz}$ and an emission sequence with eight emissions corresponds to an averaging period of $51.2 \mathrm{~ms}$. However, this average is updated each time new cross-correlations are made, yielding an update rate of $312.5 \mathrm{~Hz}$. Also, though it is possible to estimate the velocity at each image point, it would be very computational exhaustive. For this reason, the velocity was estimated at a downsampled version of the image grid. The velocity vector of each image point was then determined through spline interpolation.

\section{EMISSION SEQUENCE}

The HRVs are constructed from eight emission sources emitted using a sliding aperture of 32 elements. Each emission emits a defocused wave with an F-number of -1 , and the sources are separated linearly by $2.2 \lambda$. The transmit and receive aperture are interchanged after acquiring enough emissions to correlate two HRVs. This is because the described TVI method can only induce the transverse oscillation along one direction, which depends on the receiving aperture. It is, therefore, necessary to switch between apertures to estimate the flow in both the $x$ - and $y$-direction (see e.g., Section V). Note that the axial velocities can be estimated from both HRV pairs because the receive apodization is applied off-line. Each HRV is, thus, constructed twice, one with and one without double peak apodization.

An interleaved emission sequence is used in the acquisition, so each emission source is fired twice before emitting from a new source [41]. HRV pairs, which are separated in time by $1 / f_{p r f, e f f}=1 / f_{p r f}$, can then be formed by summing LRVs from the odd and even emissions separately. The interleaved emission sequence reduces de-correlation between the HRV pairs and allows for an increased number of emissions to form the HRV, which is especially beneficial in RCA imaging. The trade-off is that the interleaving doubles the summation period of the HRV, which enhances the motion effects. Alternatively, a non-interleaved sequence with fewer emissions could be used to avoid de-correlation at high-velocities, but this would cause a widening of the side-lobes. Optimizing the sequence to yield 
TABLE I

TVI ACQUISITION PARAMETERS

\begin{tabular}{lccc}
\hline Parameter & Value & Unit & Variable \\
\hline No. elements & $62+62$ & & \\
Center frequency & 3 & $\mathrm{MHz}$ & $f_{0}$ \\
Excitation pulse & 2 cycled sin. & & \\
Element pitch & 0.27 & $\mathrm{~mm}$ & \\
Transmit apodization & von Hann & & \\
No. active elements & 32 & & \\
F-number & -1 & & \\
Pulse repetition frequency & $10 \& 2$ & $\mathrm{kHz}$ & $f_{p r f}$ \\
Sampling frequency & 17.5 & $\mathrm{MHz}$ & \\
No. emissions & 8 & & $M$ \\
\hline
\end{tabular}

the best compromise between image quality and motion effects is a central topic for future research.

The parameters of the chosen sequence are summarized in Table I and the geometry of the emitted fields are illustrated in Fig. 5.

\section{EQUiPMENT AND METhodology}

The flow measurements were performed using a $3 \mathrm{MHz}$ $62+62$ RCA probe coupled to the experimental scanner SARUS [42]. A stationary parabolic flow was generated using a circulating flow rig with a $6 \mathrm{~mm}$ radius tube, and the volume rate was monitored using a Danfoss Magnetic flow meter MAG1100. At the measurement site of the flow rig, the probe was placed in a transducer fixture, which allows the operator to change beam-to-flow-angle, $\alpha$, and flow-rotation-angle, $\beta$. The two rotations represent the yaw and pitch-angle of the tube, and the resulting direction of the flow with respect to the probe is

$$
\vec{o}(\alpha, \beta)=\left[\begin{array}{c}
\sin \alpha \cos \beta \\
\sin \alpha \sin \beta \\
\cos \alpha
\end{array}\right] .
$$

Note that the initial orientation vector, i.e. $\vec{o}\left(0^{\circ}, 0^{\circ}\right)$, is defined to be equal to the axial unit vector. The angles were $\beta=45^{\circ}$ and $\alpha=\left(60^{\circ}, 75^{\circ}\right)$ and was chosen such that flow occurs in all three directions. The measurement setup was also recreated in a Field II simulation environment [43], [44] to investigate the performance of the estimator in response to variations in peak velocity of the flow, beam-to-flow angle and iterations of the motion compensation algorithm. The parabolic flow was simulated by moving scatterers [45], uniformly distributed within the radius of the tube, by the theoretical parabolic velocity profile:

$$
v_{\text {true }}(r)=v_{0}\left(1-\left(\frac{r}{R}\right)^{2}\right),
$$

where $v_{0}$ is the peak velocity, $R$ is the radius of the tube, and $r \in[0, R]$ denotes the radial coordinate.

Simulations were performed with an $f_{p r f}$ of $10 \mathrm{kHz}$ and measurement with an $f_{p r f}$ of $2 \mathrm{kHz}$. The ratio between $v_{0}$ and $f_{p r f}$ is, however, kept similar between simulation and measurement. The precision and accuracy of the estimator are evaluated based on the mean standard deviation, $\bar{\sigma}$, and mean bias, $B$, of the magnitude velocity profile from the axial line, which intersects the center of the vessel. In simulations, this axial line is $\vec{p}=(0,0, z)$, and in measurement, the axial line is determined from visual inspection of the B-mode image. Edge effects from the boundaries of the tube are ignored by only including the central $90 \%$ of the profile in the calculations. The following equations define the two performance metrics:

$$
\begin{gathered}
B=\frac{1}{v_{0} N} \sum_{z \in Z} v_{\text {true }}\left(z-z_{0}\right)-\bar{v}(z), \\
\bar{\sigma}=\frac{1}{v_{0} N} \sum_{z \in Z} \sqrt{\frac{1}{K-1} \sum_{i=1}^{K}\left(v_{i}(z)-\bar{v}(z)\right)^{2},} \\
\bar{v}(z)=\frac{1}{K} \sum_{i=1}^{K} v_{i}(z),
\end{gathered}
$$

where $Z \in \mathbb{R}^{N}$ is a set of axial coordinates within the central $90 \%$ of the profile, $z_{0}$ is depth of the vessel center, $v_{i}$ is the $i$ th realization of the velocity estimate and $K$ is the number of realizations of the velocity profile. In this work, the statistic of the simulated and measured data were calculated from $K=$ 150 simulated- and $K=400$ measured-realizations.

Lastly, stationary echo-canceling was applied by subtracting the mean HRV from each individual HRV. This approach suffices due to the lack of tissue motion.

\section{RESULTS}

Fig. 6 shows $B$ and $\bar{\sigma}$ against $v_{0}$, with and without motion compensation for simulated data. It is worth noting that the results presented in [23] is equivalent to the results without motion compensation given in this work. The result shows that without motion compensation bias will increase linearly with $v_{0}$. With motion compensation the bias is kept constant for most values of $v_{0}$. At $\alpha=60^{\circ}$, rapid changes in $B$ first occur beyond $v_{0}=100 \mathrm{~cm} / \mathrm{s}$ and at the lower velocities the magnitude of $B$ only slightly increases from $4.2 \%$ at $v_{0}=10$ $\mathrm{cm} / \mathrm{s}$ to $6.9 \%$ at $v_{0}=100 \mathrm{~cm} / \mathrm{s}$. The largest reduction in bias between using and not using motion compensation, at $\alpha=60^{\circ}$, is found at $v_{0}=125 \mathrm{~cm} / \mathrm{s}$, where the difference is $22.1 \%$. At $\alpha=75^{\circ}$ the motion compensation manages to keep the $B$ constant $(7.0 \% \pm 0.5 \%)$ through all the values of $v_{0}$. The largest increase in performance from using motion compensation is seen at $v_{0}=150 \mathrm{~cm} / \mathrm{s}$ where the difference in bias is $16.2 \%$. Conversely, $\bar{\sigma}$, seems to increase slightly when applying the motion compensation at the higher values of $v_{0}$. At $\alpha=60^{\circ}$, the maximum increase was found at $v_{0}=125 \mathrm{~cm} / \mathrm{s}$, where $\bar{\sigma}$ was increased from $2.2 \%$ to $4.7 \%$. At $\alpha=75^{\circ}$ the differences between $\bar{\sigma}$ when using and not using motion compensation is relatively negligible with the largest difference being $0.5 \%$ at $v_{0}=100 \mathrm{~cm} / \mathrm{s}$. The increase in $\bar{\sigma}$ is, however, a small trade-off considering the large reduction in bias. When comparing the results from the motion compensation across $\alpha=60^{\circ}$ and $\alpha=75^{\circ}$, it is clear that the latter yields the better results. This suggest that axial velocity is still the primary limiting factor of the estimator. 

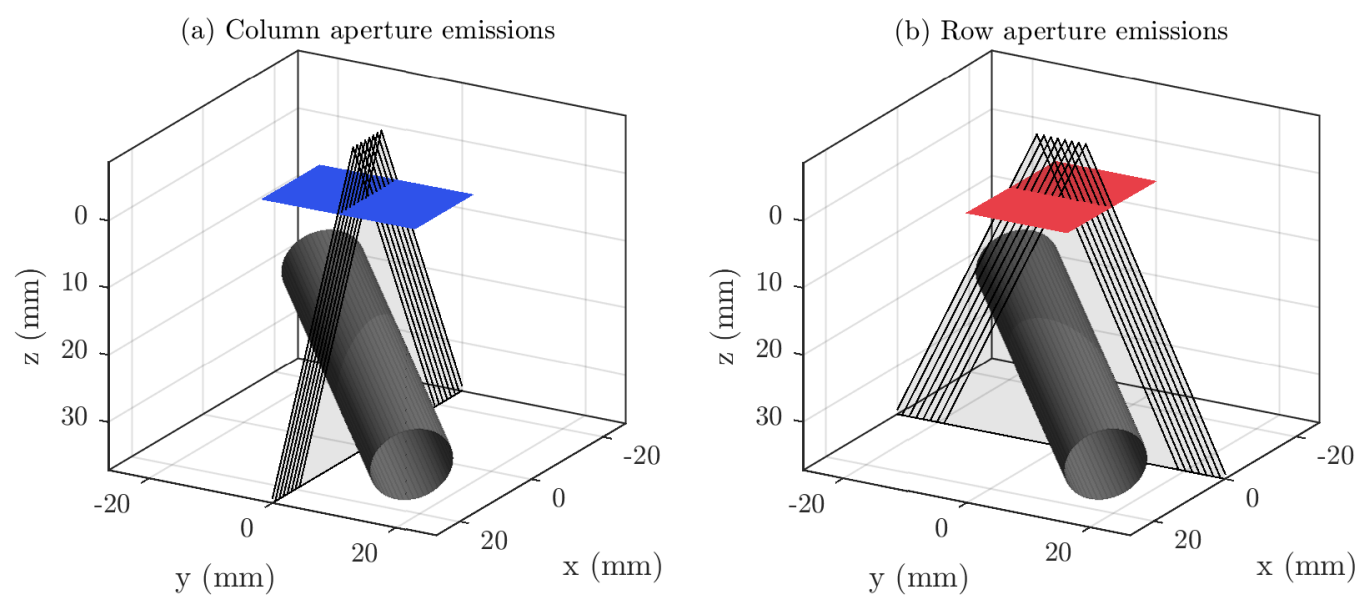

Fig. 5. Geometry of the emission sequence. Blue and red squares show the dimensions of the column- and row aperture. The cylinder indicates a vessel carrying the flow, and the outlined triangles show the emitted fields from eight emission sources.

Overall, the result shows that motion compensation manages to make the performance of the estimator less reliant on the axial velocity component's magnitude.

Fig. 7 demonstrates how the compensation reduces the motion effects in the estimates. The figure shows three 3D flow fields in a cross a vessel with a $\alpha=75^{\circ}$ with $v_{0}=125 \mathrm{~cm} / \mathrm{s}$. The first (a) shows the ideal flow field, i.e., the velocity profile used in the simulation. The second flow field (b) shows the average velocity estimate, without using motion compensation, and the third flow field (c) shows the average velocity estimate when motion compensation is applied. Without motion compensation, the entire profile is skewed both to the left and right. This is due to the deformation caused by axial motion described in Section III. The motion compensation largely removes this deformation, and the estimated flow field resembles the ideal field.

Fig. 8 demonstrates the rate of convergence of the motion compensation algorithm. The figure shows how $B$ and $\bar{\sigma}$, from the $v_{0} \geq 100 \mathrm{~cm} / \mathrm{s}$ estimates, change over the course of ten iterations. Most setups converge after three iterations and peaks in performance after two iterations. The estimates of flow at $\alpha=60^{\circ}$ and $v_{0}=125 \mathrm{~cm} / \mathrm{s}$ and $v_{0}=150 \mathrm{~cm} / \mathrm{s}$ deviate from this trend. Here, $B$ and $\bar{\sigma}$ have a slow convergence which suggest that the rate of convergence depends on the severity of the motion effects. It should be noted that the results shown in Fig. 6, 7 and 8 were acquired using three iterations of the algorithm.

Velocity profiles of the measured results are shown in Fig. 9. The peak velocity was set to $10 \mathrm{~cm} / \mathrm{s}$ and $25 \mathrm{~cm} / \mathrm{s}$ and measured at an $f_{p r f}=2 \mathrm{kHz}$. Note that the ratio between $f_{p r f}$ and $v_{0}$ is equal to the simulations with $v_{0}=50 \mathrm{~cm} / \mathrm{s}$ and $v_{0}=125 \mathrm{~cm} / \mathrm{s}$. These simulation results are therefore directly comparable to the measured data. The measured results show a similar improvement in performance in $B$ after applying motion compensation. At $\alpha=60^{\circ}$ the magnitude of $B$ is reduced by $6.4 \%$ and $18.2 \%$ for $v_{0}=10 \mathrm{~cm} / \mathrm{s}$ and $v_{0}=25$ $\mathrm{cm} / \mathrm{s}$. At $\alpha=75^{\circ}$ this magnitude reduction of $B$ is $1.1 \%$ and $9.2 \%$. The comparable simulations shows a similar reduction in bias; at $\alpha=60^{\circ}$ the reduction was found to be $6.2 \%$ and $22.1 \%$, and at $\alpha=75^{\circ}$ the bias difference was found to be $-0.3 \%$ and $11.4 \%$. Unlike the simulation results, $\bar{\sigma}$ showed small reductions or little to no difference, when applying the motion compensation.

\section{Discussion}

The motion compensation algorithm managed to reduce the TVI estimates' bias by up to $22.1 \%$ (see Fig. 6) in simulations and by up to $18.2 \%$ in measurements (see Fig. 9). The reduction in bias is achieved by reducing the deformity caused by the motion effects, as shown in Fig. 7. Note that the estimator still estimate the higher velocities without motion compensation, just in an incorrect position. This suggests the limiting factor is to a higher degree set by the imaging and, to a lesser degree, set by the cross-correlation estimator. This is further substantiated by the fact that the cross-correlation estimator performs better when the axial velocity component is lower, i.e., when $\alpha$ is closer to $90^{\circ}$ since here motion effects are less severe. For this reason, it is recommended that future work is directed towards optimizing the imaging sequence or developing new non-linear RCA imaging methods, which do not have the same constraints as the current approach. That said, the estimator's performance could be improved by adding another estimation stage, with directional beamforming, similar to the approach presented by Jensen et. al. [46]. It might also be worthwhile to explore RCA TVI methods, which can estimate all three velocity components without interchanging the receive and transmit aperture. This would effectively double the TVI frame rate as well as increase design flexibility. TVI methods that could achieve this include the 3-D speckle-tracking estimator [25] and the directional beamforming approach.

Simulations showed that motion compensation caused a slight increase in standard deviation for the higher peak velocities when $\alpha=60^{\circ}$ (see Fig. 6). This is not entirely unexpected because the input to the motion compensation algorithm, i.e., the previous iteration of the velocity estimation, 

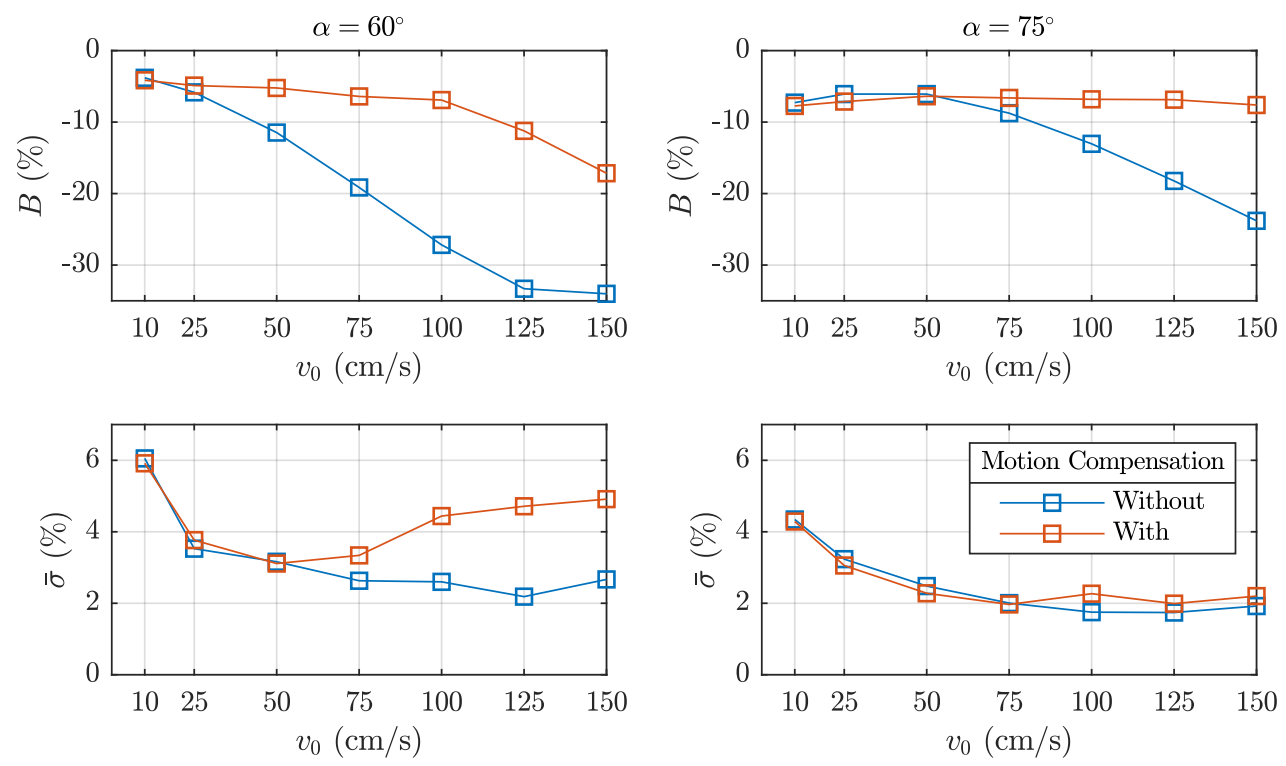

Fig. 6. Bias $(B)$ and standard deviation $\bar{\sigma}$ against peak velocity $\left(v_{0}\right)$ for simulated data. Left column shows the performance at $\alpha=60^{\circ}$ and the right column shows the performance at $\alpha=75^{\circ}$. Blue line: performance without motion compensation. Green-line: performance when using motion compensation.

(a) Target estimation

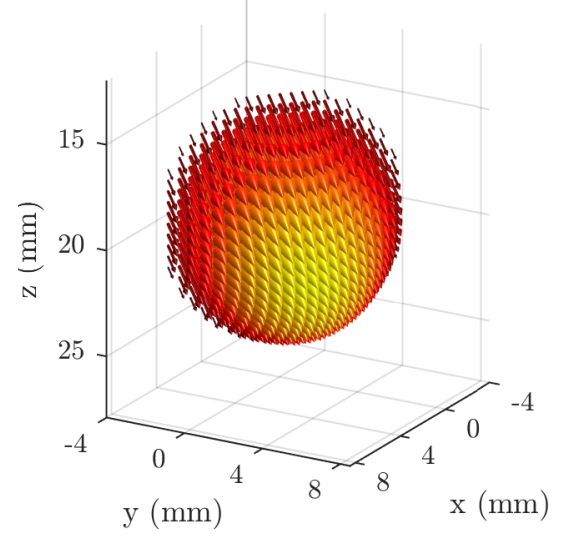

(b) Without motion comp.

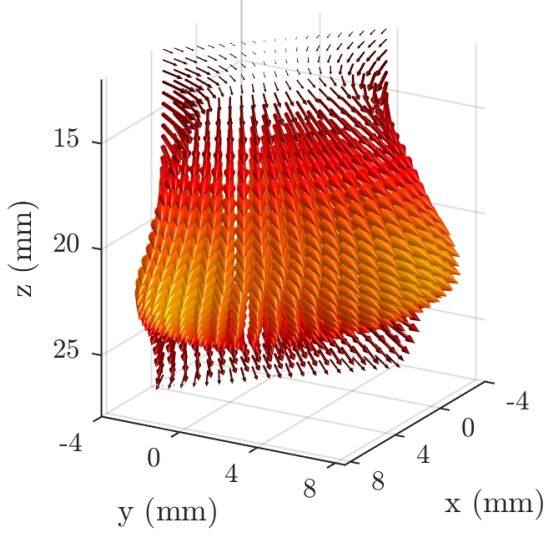

(c) With motion comp.

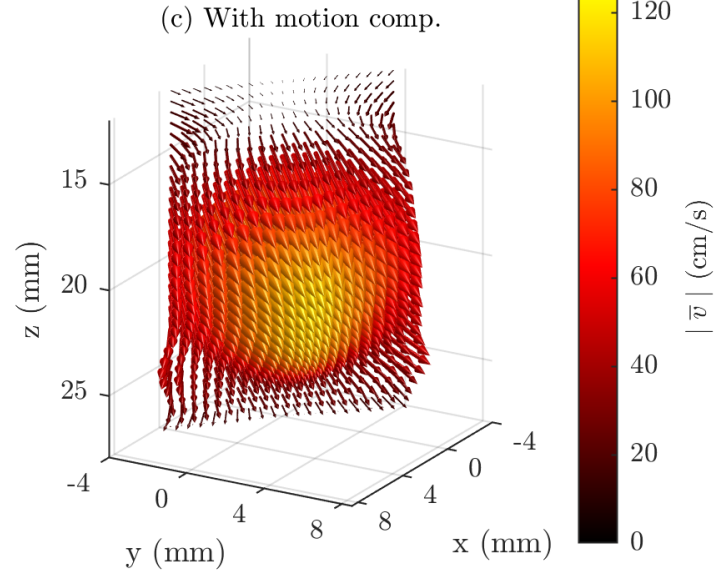

Fig. 7. TVI in a cross-section of the vessel. (a) True velocity values. (b) estimated velocity without motion compensation. (c) estimated velocity with motion compensation.

also varies. Therefore, the output variation is the result of both the variation from the velocity estimator and the variation of previous estimates. The increase in standard deviation is, however, small compared to the reduction of bias.

The increase in standard deviation is, however, not found in measured results. Here the motion compensation caused either a negligible difference in standard deviation or a slight performance increase. One explanation is that motion compensation improves signal-to-noise ratio [30], thus lowering the noise component's variation. Since the simulations do not model noise phenomena such as clutter and electrical interference, the enhanced coherence from motion compensation does not improve SNR. Therefore the performance increase from lowering the variation caused by noise is not realized in the simulations. This is believed to cause the discrepancies between the measurements and simulations.

In future work, it would be worthwhile to investigate the approach on more complex flow structures. The approach relies on reasonable initial estimates to effectively reduce the motion effects. However, these estimates are challenging to obtain if the flow field is rapidly varying. The motion effects could also cause velocities with entirely different directions to merge at one location, and in this case, it is unclear whether the actual velocity from this location can be recovered.

\section{Conclusion}

The 3-D motion compensation algorithm provides a significant bias reduction of the row-column based TVI estimator and allows the correct velocities to be estimated over a range of peak velocities and beam-to-flow angles. The 

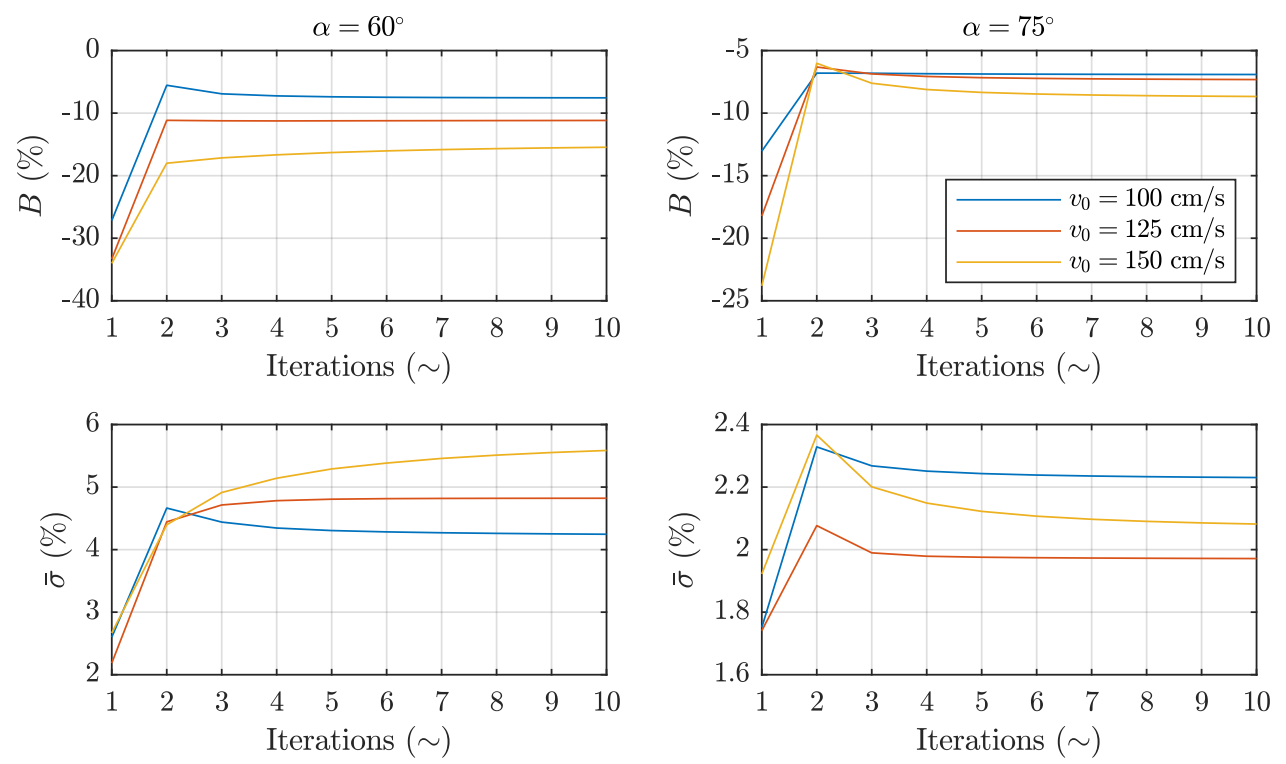

Fig. 8. Bias $(B)$ and standard deviation $\bar{\sigma}$ against iterations of the motion compensation algorithm. The performance is calculated from the estimates where $v_{0} \geq 100 \mathrm{~cm} / \mathrm{s}$. Left column shows the performance at $\alpha=60^{\circ}$ and right column shows the performance at $\alpha=75^{\circ}$.
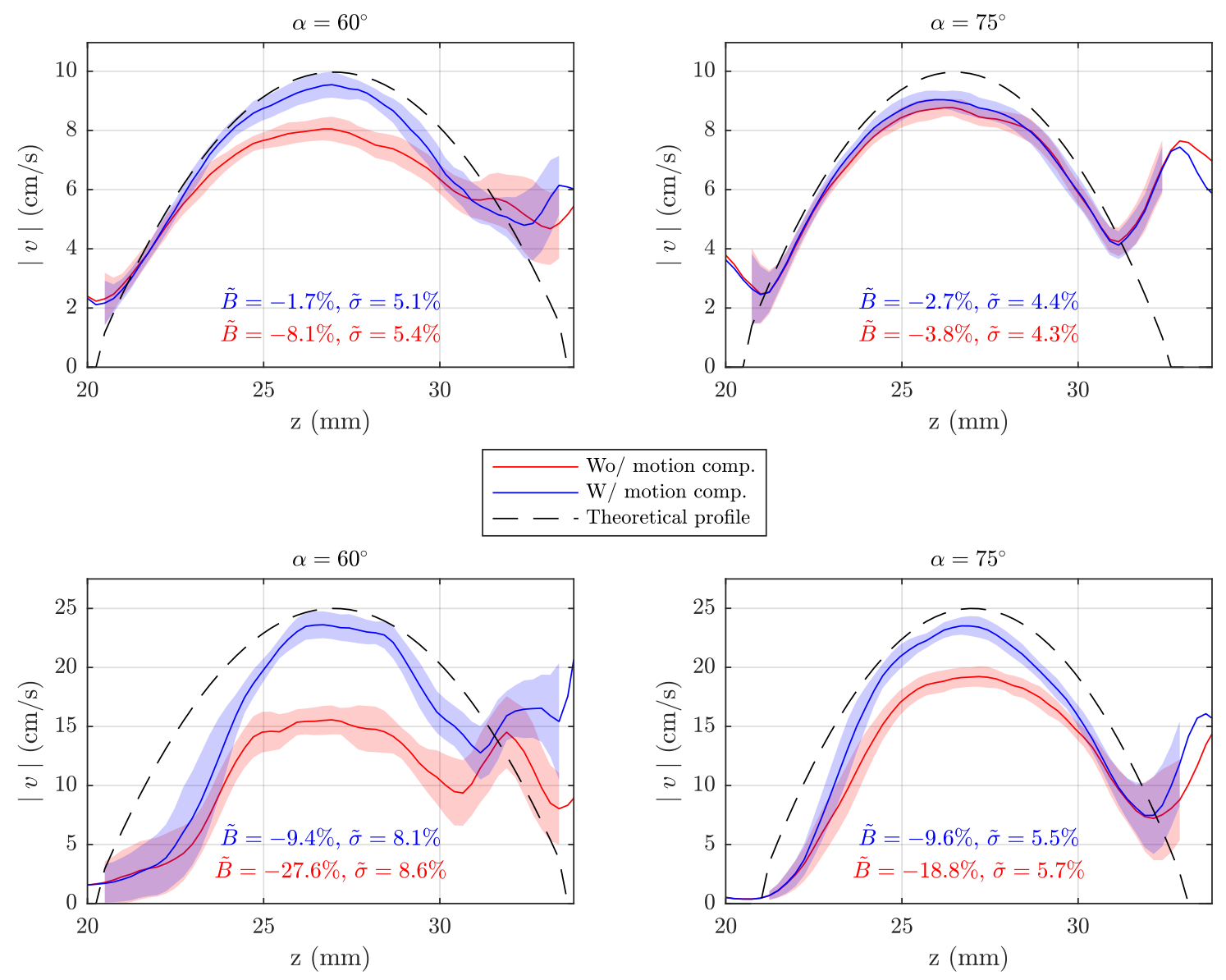

Wo/ motion comp.

$\mathrm{W} /$ motion comp.

- Theoretical profile

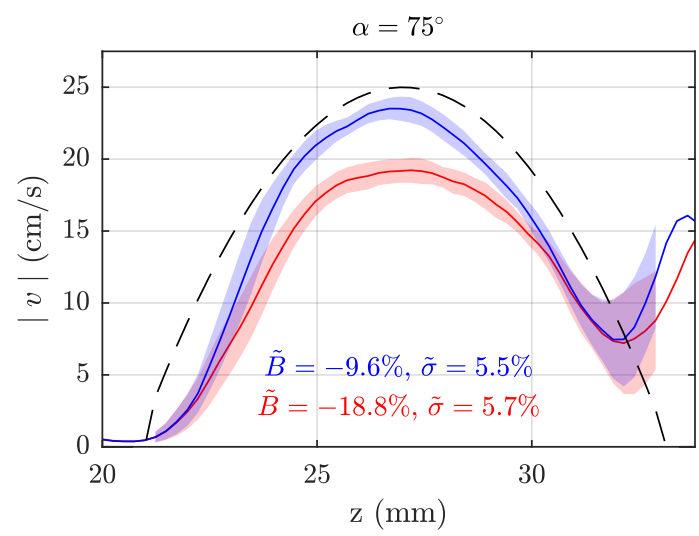

Fig. 9. Magnitude profiles from measurements of flow with $\alpha=60^{\circ}$ and $\alpha=75^{\circ}$. First row shows estimates of flow with $v_{0}=10 \mathrm{~cm} / \mathrm{s}$ and the second row shows flow estimates with $v_{0}=25 \mathrm{~cm} / \mathrm{s}$. Red line: Average estimated profile without motion compensation. Blue line: Average estimated profile with motion compensation. Shaded area: \pm one standard deviation. Dashed line: Theoretical velocity profile. 
largest bias reduction was found to be $22.1 \%$ in simulations and $18.2 \%$ in measurements. Simulations showed that the motion compensation algorithm causes a minor increase in the standard deviation at high velocities, and the highest increase was found to be $2.5 \%$. However, results from measurements showed a negligible difference in standard deviation or a slight performance increase when using motion compensation. Nevertheless, the substantial reduction in bias and the velocity-dependent skewing indicate that motion compensation should be included in SA velocity estimation with row-column probes.

\section{ACKNOWLEDGEMENT}

This work was financially supported by grant 82-2014-4 from the Danish National Advanced Technology Foundation, by grant 7050-00004B from Innovation Fund Denmark, and from BK Medical, Herlev, Denmark.

\section{REFERENCES}

[1] B. Dunmire, K. W. Beach, K.-H. Labs., M. Plett, and D. E. Strandness, "Cross-beam vector Doppler ultrasound for angle independent velocity measurements," Ultrasound Med. Biol., vol. 26, pp. 1213-1235, 2000.

[2] L. N. Bohs, B. J. Geiman, M. E. Anderson, S. C. Gebhart, and G. E. Trahey, "Speckle tracking for multi-dimensional flow estimation," Ultrasonics, vol. 38, pp. 369-375, 2000.

[3] J. A. Jensen and P. Munk, "A new method for estimation of velocity vectors," IEEE Trans. Ultrason., Ferroelec., Freq. Contr., vol. 45, no. 3, pp. 837-851, 1998.

[4] K. L. Hansen, H. Møller-Sørensen, J. Kjaergaard, J. T. Lund, M. M. Pedersen, T. Lange, J. A. Jensen, and M. B. Nielsen, "Analysis of systolic backflow and secondary helical blood flow in the ascending aorta using vector flow imaging," Ultrasound Med. Biol., vol. 42, no. 4, pp. 899-908, 2016.

[5] J. Jensen, J. B. Olesen, M. B. Stuart, P. M. Hansen, M. B. Nielsen, and J. A. Jensen, "Vector velocity volume flow estimation: Sources of error and corrections applied for arteriovenous fistulas," Ultrasonics, vol. 70, pp. 136-146, 2016.

[6] J. B. Olesen, M. S. Traberg, M. J. Pihl, and J. A. Jensen, "Noninvasive esimation of 2-D pressure gradients in steady flow using ultrasound," IEEE Trans. Ultrason., Ferroelec., Freq. Contr., vol. 61, no. 8, pp. 1409 $1418,2014$.

[7] S. W. Smith, H. G. Pavy, and O. T. von Ramm, "High speed ultrasound volumetric imaging system - Part I: Transducer design and beam steering," IEEE Trans. Ultrason., Ferroelec., Freq. Contr., vol. 38, pp. 100-108, 1991 .

[8] O. T. von Ramm, S. W. Smith, and H. G. Pavy, "High speed ultrasound volumetric imaging system - Part II: Parallel processing and image display," IEEE Trans. Ultrason., Ferroelec., Freq. Contr., vol. 38, pp. 109$115,1991$.

[9] D. H. Turnbull and F. S. Foster, "Beam steering with pulsed twodimensional transducer arrays," IEEE Trans. Ultrason., Ferroelec., Freq. Contr., vol. 38, pp. 320-333, July 1991.

[10] G. R. Lockwood, J. R. Talman, and S. S. Brunke, "Real-time 3-D ultrasound imaging using sparse synthetic aperture beamforming," IEEE Trans. Ultrason., Ferroelec., Freq. Contr., vol. 45, pp. 980-988, 1998.

[11] C. E. Morton and G. R. Lockwood, "Theoretical assessment of a crossed electrode 2-D array for 3-D imaging," in Proc. IEEE Ultrason. Symp., pp. 968-971, 2003.

[12] C. E. M. Démoré, A. W. Joyce, K. Wall, and G. R. Lockwood, "Realtime volume imaging using a crossed electrode array," IEEE Trans. Ultrason., Ferroelec., Freq. Contr., vol. 56, no. 6, pp. 1252-1261, 2009.

[13] S. Blaak, Z. Yu, G. C. M. Meijer, C. Prins, C. T. Lancee, J. G. Bosch, and N. de Jong, "Design of a micro-beamformer for a 2D piezoelectric ultrasound transducer," in Proc. IEEE Ultrason. Symp., pp. 1338-1341, sep 2009.

[14] P. Mattesini, A. Ramalli, L. Petrusca, O. Basset, H. Liebgott, and P. Tortoli, "Spectral doppler measurements with 2-d sparse arrays," IEEE Trans. Ultrason., Ferroelec., Freq. Contr., vol. 67, no. 2, pp. 278-285, 2020.
[15] H. Yoon and T. K. Song, "Sparse rectangular and spiral array designs for 3-D medical ultrasound imaging," Sensors, vol. 20, no. 1, p. 173, 2020.

[16] P. Santos, G. U. Haugen, L. Løvstakken, E. Samset, and J. D’hooge, "Diverging wave volumetric imaging using subaperture beamforming," IEEE Trans. Ultrason., Ferroelec., Freq. Contr., vol. 63, no. 12, pp. 2114-2124, 2016

[17] S. Rossi, A. Ramalli, F. Fool, and P. Tortoli, "High-frame-rate 3-d vector flow imaging in the frequency domain," Applied Sciences, vol. 10, no. 15 , p. $5365,2020$.

[18] M. Correia, J. Provost, M. Tanter, and M. Pernot, "In-vivo 4D ultrafast vector flow imaging: quantitative assessment of arterial blood flow," in Proc. IEEE Ultrason. Symp., pp. 1-4, 2016.

[19] M. Flesch, M. Pernot, J. Provost, G. Ferin, A. Nguyen-Dinh, M. Tanter, and T. Deffieux, "4D in vivo ultrafast ultrasound imaging using a rowcolumn addressed matrix and coherently-compounded orthogonal plane waves," Phys. Med. Biol., vol. 62, pp. 4571-4588, 2017.

[20] S. Harput, K. Christensen-Jeffries, A. Ramalli, J. Brown, J. Zhu, G. Zhang, C. H. Leow, M. Toulemonde, E. Boni, P. Tortoli, R. J. Eckersley, C. Dunsby, and M. Tang, "3-D super-resolution ultrasound (SR-US) imaging with a 2-D sparse array," arXiv preprint, p. 1902.01608v1, 2019.

[21] J. Sauvage, M. Flesch, G. Ferin, A. Nguyen-Dinh, J. Poree, M. Tanter, M. Pernot, and T. Deffieux, "A large aperture row column addressed probe for in vivo $4 \mathrm{~d}$ ultrafast doppler ultrasound imaging," Phys. Med. Biol., vol. 63, pp. 1-12, 2018.

[22] L. Petrusca, F. Varray, R. Souchon, A. Bernard, J. Y. Chapelon, H. Liebgott, W. A. N'Djin, and M. Viallon, "Fast volumetric ultrasound B-mode and Doppler imaging with a new high-channels density platform for advanced 4D cardiac imaging/therapy," Applied Sciences (Switzerland), vol. 8, no. 2, pp. 200:1-15, 2018.

[23] M. Schou, L. Jørgensen, C. Beers, M. S. Traberg, B. G. Tomov, M. B. Stuart, and J. A. Jensen, "Fast 3-D Velocity Estimation in 4-D using a 62 + 62 Row-Column Addressed Array," IEEE Trans. Ultrason., Ferroelec. Freq. Contr., vol. -, no. -, pp. -, 2020.

[24] S. Holbek, T. L. Christiansen, M. Engholm, A. Lei, M. B. Stuart, C. Beers, L. N. Moesner, J. P. Bagge, E. V. Thomsen, and J. A. Jensen, "3-D vector flow using a row-column addressed CMUT array," in Proc. SPIE Med. Imag., vol. 9790, pp. 979005-979005-8, 2016.

[25] M. S. Wigen, S. Fadnes, A. Rodriguez-Molares, T. Bjåstad, M. Eriksen, K. H. Stensæth, A. Støylen, and L. Løvstakken, "4-D intracardiac ultrasound vector flow imaging-reasibility and comparison to phasecontrast MRI," IEEE Trans. Med. Imag., vol. 37, pp. 2619-2629, December 2018

[26] G. E. Trahey and L. F. Nock, "Synthetic receive aperture imaging with phase correction for motion and for tissue inhomogenities - part II: effects of and correction for motion," IEEE Trans. Ultrason., Ferroelec., Freq. Contr., vol. 39, pp. 496-501, 1992.

[27] M. Karaman and M. O'Donnell, "Subaperture processing for ultrasonic imaging," IEEE Trans. Ultrason., Ferroelec., Freq. Contr., vol. 45, pp. 126-135, 1998.

[28] C. R. Hazard and G. R. Lockwood, "Theoretical assessment of a synthetic aperture beamformer for real-time 3-D imaging," IEEE Trans. Ultrason., Ferroelec., Freq. Contr., vol. 46, pp. 972-980, 1999.

[29] J. S. Jeong, J. S. Hwang, M. H. Bae, and T. K. Song, "Effects and limitations of motion compensation in synthetic aperture techniques," in Proc. IEEE Ultrason. Symp., vol. 2, pp. 1759-1762, 2000.

[30] K. S. Kim, J. S. Hwang, J. S. Jeong, and T. K. Song, "An efficient motion estimation and compensation method for ultrasound synthetic aperture imaging," Ultrason. Imaging, vol. 24, pp. 91-99, 2002.

[31] S. I. Nikolov and J. A. Jensen, "In-vivo synthetic aperture flow imaging in medical ultrasound," IEEE Trans. Ultrason., Ferroelec., Freq. Contr., vol. 50, no. 7, pp. 848-856, 2003.

[32] N. Oddershede and J. A. Jensen, "Effects influencing focusing in synthetic aperture vector flow imaging," IEEE Trans. Ultrason., Ferroelec., Freq. Contr., vol. 54, no. 9, pp. 1811-1825, 2007.

[33] B. Denarie, T. A. Tangen, I. K. Ekroll, N. Rolim, H. Torp, T. Bjastad, and L. Løvstakken, "Coherent plane wave compounding for very high frame rate ultrasonography of rapidly moving targets," IEEE Trans. Ultrason., Ferroelec., Freq. Contr., vol. 32, no. 7, pp. 1265-1276, 2013.

[34] K. L. Gammelmark and J. A. Jensen, "2-D tissue motion compensation of synthetic transmit aperture images," IEEE Trans. Ultrason., Ferroelec., Freq. Contr., vol. 61, pp. 594-610, April 2014.

[35] I. K. Ekroll, M. M. Voormolen, O. K. Standal, J. M. Rau, and L. Løvstakken, "Coherent compounding in doppler imaging," IEEE Trans. Ultrason., Ferroelec., Freq. Contr., vol. 62, no. 9, pp. 1634-1643, 2015. 
[36] L. T. Jørgensen, M. Schou, M. B. Stuart, and J. A. Jensen, "Tensor velocity imaging with motion correction," in Proc. IEEE Ultrason. Symp., pp. 1-4, 2020.

[37] M. F. Rasmussen, T. L. Christiansen, E. V. Thomsen, and J. A Jensen, "3-D imaging using row-column-addressed arrays with integrated apodization - Part I: Apodization design and line element beamforming," IEEE Trans. Ultrason., Ferroelec., Freq. Contr., vol. 62, no. 5, pp. 947-958, 2015.

[38] F. L. Thurstone and O. T. von Ramm, "A new ultrasound imaging technique employing two-dimensional electronic beam steering," in Acoustical Holography (P. S. Green, ed.), vol. 5, (New York), pp. 249259, Plenum Press, 1974.

[39] J. A. Jensen, "Estimation of high velocities in synthetic aperture imaging: II: Experimental investigation," IEEE Trans. Ultrason., Ferroelec., Freq. Contr., vol. 66, no. 6, pp. 1032-1038, 2019.

[40] S. G. Foster, A pulsed ultrasonic flowmeter employing time domain methods. PhD thesis, Dept. Elec. Eng., University of Illinois, Urbana, Ill., 1985.

[41] J. A. Jensen, "Estimation of high velocities in synthetic aperture imaging: I: Theory," IEEE Trans. Ultrason., Ferroelec., Freq. Contr., vol. 66, no. 6, pp. 1024-1031, 2019.

[42] J. A. Jensen, H. Holten-Lund, R. T. Nilsson, M. Hansen, U. D. Larsen, R. P. Domsten, B. G. Tomov, M. B. Stuart, S. I. Nikolov, M. J. Pihl, Y. Du, J. H. Rasmussen, and M. F. Rasmussen, "SARUS: A synthetic aperture real-time ultrasound system," IEEE Trans. Ultrason., Ferroelec., Freq. Contr, vol. 60, no. 9, pp. 1838-1852, 2013.

[43] J. A. Jensen, "Field: A program for simulating ultrasound systems," Med. Biol. Eng. Comp., vol. 10th Nordic-Baltic Conference on Biomedical Imaging, Vol. 4, Supplement 1, Part 1, pp. 351-353, 1996.

[44] J. A. Jensen and N. B. Svendsen, "Calculation of pressure fields from arbitrarily shaped, apodized, and excited ultrasound transducers," IEEE Trans. Ultrason., Ferroelec., Freq. Contr., vol. 39, no. 2, pp. 262-267, 1992.

[45] J. A. Jensen and P. Munk, "Computer phantoms for simulating ultrasound B-mode and CFM images," in Acoustical Imaging (S. Lees and L. A. Ferrari, eds.), vol. 23, pp. 75-80, 1997.

[46] J. Jensen, C. A. Villagomez-Hoyos, M. B. Stuart, C. Ewertsen, M. B. Nielsen, and J. A. Jensen, "Fast plane wave 2-D vector flow imaging using transverse oscillation and directional beamforming," IEEE Trans. Ultrason., Ferroelec., Freq. Contr., vol. 64, no. 7, pp. 1050-1062, 2017.

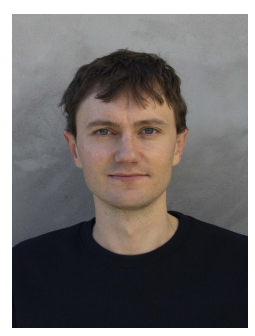

Lasse Thurmann Jørgensen received the M.S.c. Degree in biomedical engineering from the Technical University of Denmark, Lyngby, Denmark, in 2019. He is currently a Ph.D. candidate at DTU Health Tech in the Center for Fast Ultrasound imaging, where his topic of research includes motion estimation with 3-D ultrasound systems.

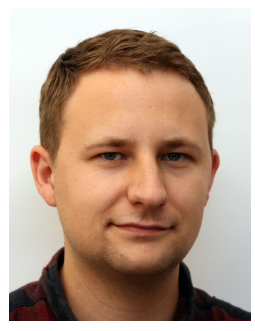

Mikkel Schou received the M.Sc. degree in biomedical engineering from the Technical University of Denmark, Kongens Lyngby, Denmark, and the University of Copenhagen, Copenhagen, Denmark, in 2017. He is currently pursuing a Ph.D. degree in biomedical engineering with the Center for Fast Ultrasound Imaging, Technical University of Denmark. The topic of his Ph.D. research is 3-D ultrasound perfusion and flow imaging using row-column arrays.

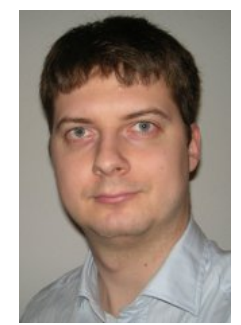

Matthias Bo Stuart received the M.Sc. and Ph.D. degrees in Computer Engineering from the Technical University of Denmark, Lyngby, Denmark, in 2006 and 2010, respectively. He is currently an Associate Professor with the Biomedical Engineering Section, Department of Health Technology at the Technical University of Denmark. His research interests include synthetic aperture methods for both anatomical and flow imaging in both 2-D and 3-D, ultrasound systems, and real-time implementations of ultrasound processing algorithms.

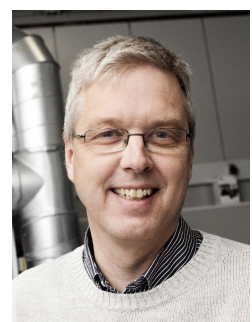

Jørgen Arendt Jensen (M'93-SM'02-F'12) received the M.Sc. degree in 1985, the Ph.D. degree in 1989, and the Dr.Techn. degree in 1996, all from the Technical University of Denmark. Since 1993, he has been a Full Professor of Biomedical Signal Processing with the Department of Health Technology, Technical University of Denmark. He has been the founder and head of the Center for Fast Ultrasound Imaging since its inauguration in 1998. CFU has contributed with innovations in transverse oscillation vector flow imaging, synthetic aperture flow imaging in 2-D and 3-D, ultrasound simulation, research scanners, and row-column probes and beamforming. He has published more than 500 journal and conference papers on signal processing and medical ultrasound and the book Estimation of Blood Velocities Using Ultrasound (Cambridge Univ. Press), 1996. He is also the developer and maintainer of the Field II simulation program. He has been a visiting scientist at Duke University, Stanford University, and the University of Illinois at Urbana-Champaign. He was the founder and head of the Biomedical Engineering group from 2007 to 2010. In 2003, he was one of the founders of the biomedical engineering program in Medicine and Technology, which is a joint degree program between the Technical University of Denmark and the Faculty of Health and Medical Sciences at the University of Copenhagen. The degree is one of the most sought-after engineering degrees in Denmark. He was chairman of the study board from 2003 to 2010 and Adjunct Professor with the University of Copenhagen from 2005 to 2010. He has given several short courses on simulation, synthetic aperture imaging, and flow estimation at international scientific conferences and teaches biomedical signal processing and medical imaging at the Technical University of Denmark. His research is centered around the simulation of ultrasound imaging, synthetic aperture imaging, vector blood flow estimation, 3-D and super-resolution imaging, row-column probes, and the construction of ultrasound research systems. 